\title{
Mechanical Properties of a Calcium Dietary Supplement, Calcium Fumarate Trihydrate
}

Shijing Sun ${ }^{a}$, Sebastian Henke ${ }^{\mathrm{a}, b}$, Michael T. Wharmby ${ }^{\mathrm{a}, c}$, Hamish H.-M. Yeung ${ }^{d}$, Wei $\mathrm{Li}^{a_{*}, \mathrm{e}}$ and Anthony K. Cheetham ${ }^{a_{*}}$

${ }^{a}$ Department of Materials Science and Metallurgy, University of Cambridge, Cambridge CB3 OFS, U.K.

${ }^{b}$ Lehrstuhl für Anorganische Chemie II, Ruhr-Universität Bochum, 44780 Bochum, Germany.

${ }^{c}$ Diamond Light Source Ltd., Diamond House, Harwell Science \& Innovation Campus, Didcot, Oxforshire, OX11 ODE, U.K.

${ }^{d}$ International Center for Young Scientists (ICYS), International Center for Materials Nanoarchitectonics (MANA), National Institute for Materials Science (NIMS), Namiki 1-1, Tsukuba, Ibaraki 305-0044, Japan

${ }^{e}$ School of Physics and Wuhan National High Magnetic Field Center, Huazhong University of Science and Technology, Wuhan 430074, China. 


\section{SUPPORTING INFORMATION}

Table S1. O-H $\cdots$ O hydrogen bond distances $(\AA)$ and angles, $<(X H Y)\left({ }^{\circ}\right)$ in CFT at ambient conditions and their projections along the principle cell directions.

\begin{tabular}{|c|c|c|c|c|c|c|c|}
\hline O-H•••O & $\begin{array}{c}d \\
(\mathbf{X} \bullet \bullet H)\end{array}$ & $d(\mathrm{H} \bullet \bullet \bullet)$ & $d(\mathrm{X} \bullet \bullet Y)$ & $<($ XHY $)$ & $d_{a}$ & $d_{b}$ & $d_{c}$ \\
\hline O6W-H6wa $\bullet • O 3^{1}$ & 0.85 & $1.96(2)$ & $2.779(3)$ & $161(3)$ & $2.184(3)$ & $0.437(2)$ & $1.661(3)$ \\
\hline $\mathrm{O} 7 \mathrm{~W}-\mathrm{H} 7$ wa $\bullet \bullet \mathrm{O}^{1}$ & 0.85 & $1.92(2)$ & $2.738(3)$ & $161(3)$ & $0.598(2)$ & $2.397(2)$ & $1.180(3)$ \\
\hline $\mathrm{O} 5 \mathrm{~W}-\mathrm{H} 5 \mathrm{wb} \cdot \cdots \mathrm{O}^{2}$ & 0.85 & $2.04(2)$ & $2.877(3)$ & $170(6)$ & $2.140(3)$ & $0.747(4)$ & $1.772(4)$ \\
\hline O6W-H6wa $\bullet \bullet O 4^{3}$ & 0.85 & $1.95(1)$ & $2.781(3)$ & $165(4)$ & $2.204(3)$ & $0.340(2)$ & $1.662(3)$ \\
\hline $\mathrm{O} 7 \mathrm{~W}-\mathrm{H} 7 \mathrm{wb} \cdot \cdots \mathrm{O}^{4}$ & 0.85 & $1.91(1)$ & $2.745(3)$ & $169(4)$ & $0.482(2)$ & $2.431(2)$ & $1.181(3)$ \\
\hline O5W-H5wa・••O3 ${ }^{5}$ & 0.85 & $2.09(5)$ & $2.936(4)$ & $175(4)$ & $2.248(3)$ & $0.649(4)$ & $1.773(4)$ \\
\hline
\end{tabular}

Note: $d_{\mathrm{a}}, d_{\mathrm{b}}$ and $d_{c}$ are the projections of the $\mathrm{O}_{\text {Donor }} \cdots \mathrm{O}_{\text {Acceptor }}$ vectors along the crystallographic $a, b$ and $c$ directions respectively, calculated using the orthogonal coordinates. Symmetry codes: (1) $1 / 2-x, 1 / 2+y,-1 / 2+z$; (2) $3 / 2-\mathrm{x}, 1 / 2+\mathrm{y}, 1 / 2+\mathrm{z}$; (3) 3/2-x,1/2+y,-1/2+z; (4) $1-\mathrm{x}, 1-\mathrm{y},-1 / 2+\mathrm{z}$; (5) $1 / 2-\mathrm{x},-1 / 2+\mathrm{y},-0.5+\mathrm{z}$.

Table S2. Crystallographic data of CFT as a function of pressure.

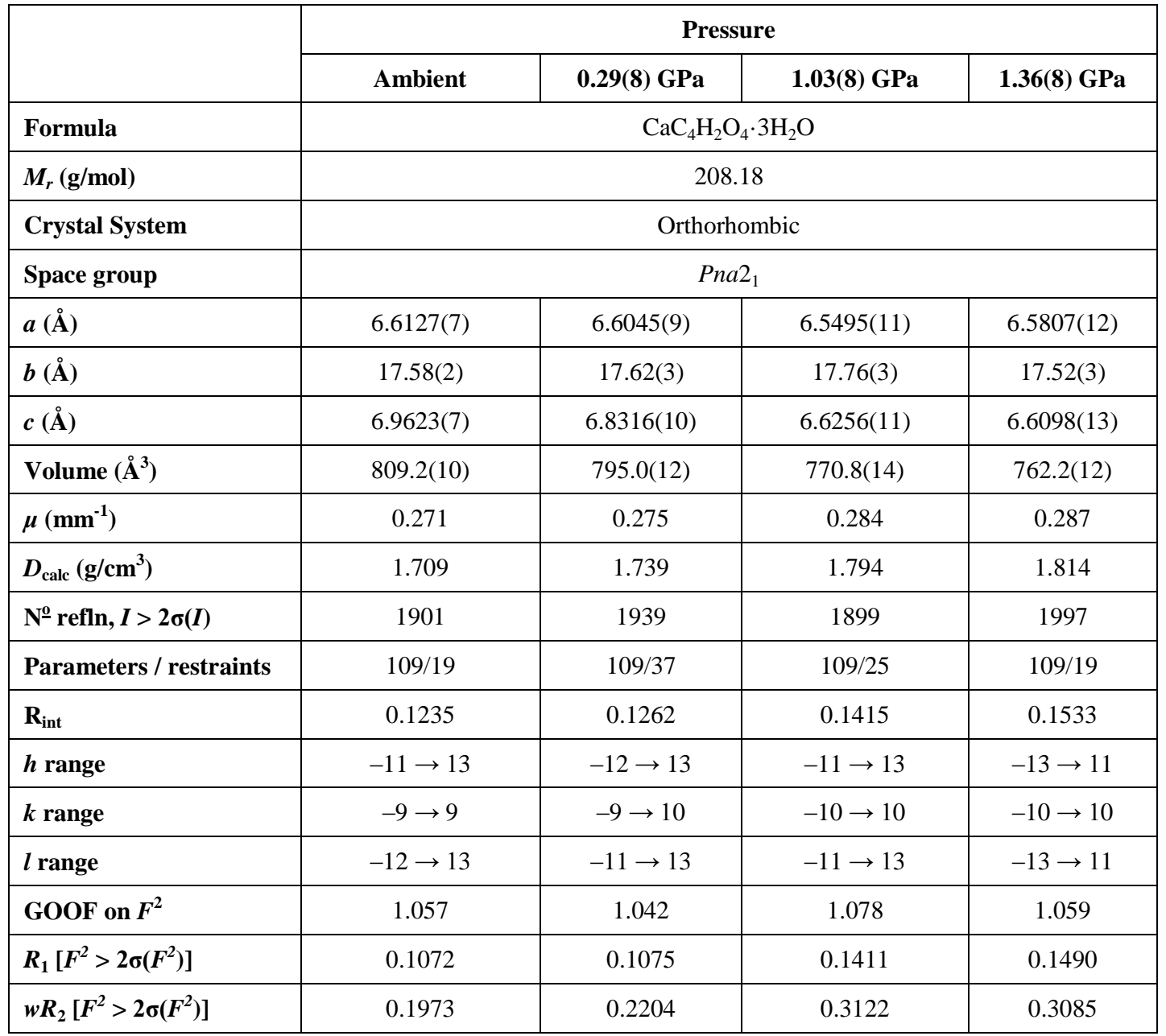


Table S3. Selected $\mathrm{Ca}-\mathrm{O}$ bond lengths $(\AA)$ within the $\mathrm{CaO}_{8}$ polyhedron in framework CFT as a function of pressure.

\begin{tabular}{|c|c|c|c|c|}
\hline \multirow{2}{*}{ Bond length } & \multicolumn{4}{|c|}{ Pressure } \\
\cline { 2 - 5 } & Ambient & $\mathbf{0 . 2 9}(\mathbf{8}) \mathbf{G P a}$ & $\mathbf{1 . 0 3}(\mathbf{8}) \mathbf{G P a}$ & $\mathbf{1 . 3 6}(\mathbf{8}) \mathbf{G P a}$ \\
\hline Ca1-O1 & $2.49(2)$ & $2.50(2)$ & $2.51(3)$ & $2.48(3)$ \\
\hline Ca1-O1 & $2.338(8)$ & $2.344(9)$ & $2.333(12)$ & $2.334(14)$ \\
\hline Ca1-O2 & $2.563(19)$ & $2.56(2)$ & $2.56(3)$ & $2.53(3)$ \\
\hline Ca1-O2 & $2.341(8)$ & $2.336(10)$ & $2.319(13)$ & $2.326(13)$ \\
\hline Ca1-O5w & $2.372(13)$ & $2.353(12)$ & $2.34(2)$ & $2.33(2)$ \\
\hline Ca1-O6w & $2.38(2)$ & $2.40(2)$ & $2.44(3)$ & $2.41(4)$ \\
\hline Ca1-O7w & $2.611(11)$ & $2.590(12)$ & $2.595(15)$ & $2.609(16)$ \\
\hline Ca1-O7w ${ }^{1}$ & $2.583(10)$ & $2.590(11)$ & $2.548(14)$ & $2.566(16)$ \\
\hline
\end{tabular}

Symmetry codes: (1) $-1 / 2+x, 3 / 2-y,+z ;$ (2) $1 / 2+x, 3 / 2-y,+z$.

Table S4. Selected $\mathrm{O}-\mathrm{Ca}-\mathrm{O}$ bond angles $\left({ }^{\circ}\right)$ within the $\mathrm{CaO}_{8}$ polyhedron in framework CFT as a function of pressure.

\begin{tabular}{|c|c|c|c|c|}
\hline \multirow[t]{2}{*}{ Bond angle } & \multicolumn{4}{|c|}{ Pressure } \\
\hline & Ambient & $0.29(8) \mathrm{GPa}$ & 1.03(8) GPa & 1.36(8) GPa \\
\hline $\mathrm{O} 1^{1}-\mathrm{Ca} 1-\mathrm{O} 1$ & $124.5(4)$ & $124.3(5)$ & $123.6(6)$ & $122.8(7)$ \\
\hline $\mathrm{O} 1-\mathrm{Ca} 1-\mathrm{O} 2$ & $51.4(4)$ & $51.2(4)$ & $50.5(6)$ & $51.7(6)$ \\
\hline $\mathrm{O} 1^{1}-\mathrm{Ca} 1-\mathrm{O} 2^{2}$ & $141.9(6)$ & 141.7(6) & 141.2(9) & $141.6(8)$ \\
\hline $\mathrm{O} 1^{1}-\mathrm{Ca} 1-\mathrm{O} 2$ & $75.5(6)$ & $75.6(7)$ & $75.7(9)$ & $85.0(8)$ \\
\hline $\mathrm{O} 1^{1}-\mathrm{Ca} 1-\mathrm{O} 5 \mathrm{~W}$ & $72.6(3)$ & $72.5(4)$ & $72.1(5)$ & $72.4(5)$ \\
\hline $\mathrm{O} 1^{1}-\mathrm{Ca}-\mathrm{O} 6 \mathrm{~W}$ & $85.2(5)$ & $84.2(6)$ & $83.4(8)$ & $85.0(8)$ \\
\hline $\mathrm{O} 1^{1}-\mathrm{Ca} 1-\mathrm{O} 7 \mathrm{~W}^{2}$ & $146.2(3)$ & $146.8(3)$ & $146.9(5)$ & $147.1(5)$ \\
\hline O1-Ca1-O7W & $97.8(6)$ & $98.2(7)$ & $97.0(9)$ & 99.5(9) \\
\hline $\mathrm{O} 1-\mathrm{Ca} 1-\mathrm{O} 7 \mathrm{~W}^{2}$ & $66.4(5)$ & $66.1(5)$ & $67.1(7)$ & $67.5(7)$ \\
\hline $\mathrm{O} 1^{1}-\mathrm{Ca} 1-\mathrm{O} 7 \mathrm{~W}$ & $68.1(4)$ & $68.3(4)$ & $68.9(6)$ & $68.8(6)$ \\
\hline $\mathrm{O} 2^{2}-\mathrm{Ca} 1-\mathrm{O} 1$ & $76.8(6)$ & $77.0(6)$ & $77.1(9)$ & $75.4(9)$ \\
\hline $\mathrm{O} 2^{2}-\mathrm{Ca} 1-\mathrm{O} 2$ & $126.4(4)$ & $126.4(4)$ & $125.7(6)$ & $125.5(6)$ \\
\hline $\mathrm{O} 2^{2}-\mathrm{Ca} 1-\mathrm{O} 5 \mathrm{~W}$ & 73.3(3) & $72.9(4)$ & $72.2(5)$ & $72.4(5)$ \\
\hline $\mathrm{O} 2^{2}-\mathrm{Ca} 1-\mathrm{O} 6 \mathrm{~W}$ & $85.9(5)$ & $85.5(6)$ & $85.5(8)$ & $86.0(8)$ \\
\hline $\mathrm{O} 2-\mathrm{Ca} 1-\mathrm{O} 7 \mathrm{~W}$ & $64.7(5)$ & $65.1(5)$ & $64.3(7)$ & $65.3(7)$ \\
\hline $\mathrm{O} 2^{2}-\mathrm{Ca} 1-\mathrm{O} 7 \mathrm{~W}$ & 146.3(3) & 146.3(4) & $146.9(5)$ & $146.9(5)$ \\
\hline O2-Ca1-O7W ${ }^{2}$ & $98.0(6)$ & $97.6(6)$ & 98.1(9) & 98.2(9) \\
\hline $\mathrm{O} 2^{2}-\mathrm{Ca} 1-\mathrm{O} 7 \mathrm{~W}^{2}$ & $68.2(4)$ & $68.3(4)$ & $68.5(6)$ & $68.8(6)$ \\
\hline O5W-Ca1-O1 & 98.1(7) & $98.2(7)$ & $99.3(11)$ & $98.4(11)$ \\
\hline $\mathrm{O} 5 \mathrm{~W}-\mathrm{Ca} 1-\mathrm{O} 2$ & $98.7(6)$ & 99.1(7) & $99.3(11)$ & $100.2(10)$ \\
\hline O5W-Ca1-O6W & $102.2(8)$ & $99.6(8)$ & $95.9(12)$ & $96.3(12)$ \\
\hline O5W-Ca1O7W ${ }^{2}$ & $140.8(3)$ & $140.5(3)$ & $140.5(4)$ & $140.9(5)$ \\
\hline O5W-Ca1-O7W & $140.0(3)$ & $140.3(3)$ & $140.3(4)$ & $140.1(5)$ \\
\hline O6W-Ca1-O1 & 148.1(4) & $150.0(4)$ & $152.0(5)$ & $151.5(5)$ \\
\hline O6W-Ca1-O2 & $145.8(4)$ & $146.7(4)$ & $148.0(5)$ & $147.6(5)$ \\
\hline O6W-Ca1-O7W & $82.0(6)$ & $83.0(6)$ & $85.7(8)$ & $84.3(8)$ \\
\hline O6W-Ca1-O7W ${ }^{2}$ & $82.5(6)$ & $84.9(6)$ & $86.2(8)$ & $85.9(8)$ \\
\hline $\mathrm{O}^{2} \mathrm{~W}^{2}-\mathrm{Ca} 1-\mathrm{O} 7 \mathrm{~W}$ & $79.1(2)$ & $79.2(2)$ & 79.1(3) & $79.0(4)$ \\
\hline
\end{tabular}

Symmetry codes: (1) $-1 / 2+\mathrm{x}, 3 / 2-\mathrm{y},+\mathrm{z} ;$ (2) $1 / 2+\mathrm{x}, 3 / 2-\mathrm{y},+\mathrm{z}$ 
Table S5. Selected $\mathrm{O} \cdots$ O hydrogen bond distances (in $\AA$ ) for framework CFT as a function of pressure.

\begin{tabular}{|c|c|c|c|c|}
\hline \multirow[t]{2}{*}{ Bond length } & \multicolumn{4}{|c|}{ Pressure } \\
\hline & Ambient & $0.29(8)$ GPa & $1.03(8) \mathrm{GPa}$ & $1.36(8) \mathrm{GPa}$ \\
\hline $\mathrm{O} 6 \mathrm{~W} \cdot \bullet \cdot \mathrm{O} 3^{1}$ & $2.77(1)$ & $2.78(1)$ & $2.74(2)$ & $2.74(2)$ \\
\hline O7W•••O3 ${ }^{1}$ & $2.71(3)$ & $2.68(3)$ & $2.75(4)$ & $2.68(4)$ \\
\hline $\mathrm{O} 5 \mathrm{~W} \bullet \bullet \mathrm{O}^{2}$ & $2.89(1)$ & $2.84(2)$ & $2.76(2)$ & $2.75(2)$ \\
\hline $\mathrm{O} 6 \mathrm{~W} \cdot \cdots \mathrm{O}^{3}{ }^{3}$ & $2.75(1)$ & $2.79(1)$ & $2.76(2)$ & $2.75(2)$ \\
\hline $\mathrm{O} 7 \mathrm{~W} \bullet \bullet O 4^{4}$ & $2.75(3)$ & $2.76(3)$ & $2.70(4)$ & $2.68(4)$ \\
\hline $\mathrm{O} 5 \mathrm{~W} \cdot \bullet \mathrm{O}^{5}$ & $2.92(1)$ & $2.88(1)$ & $2.83(2)$ & $2.84(2)$ \\
\hline
\end{tabular}

Symmetry codes: (1) $1 / 2-\mathrm{x}, 1 / 2+\mathrm{y},-1 / 2+\mathrm{z}$; (2) 3/2-x,1/2+y,1/2+z; (3) 3/2-x,1/2+y,-1/2+z; (4) $1-\mathrm{x}, 1-\mathrm{y},-1 / 2+\mathrm{z}$; (5) $1 / 2-\mathrm{x},-1 / 2+\mathrm{y},-0.5+\mathrm{z}$. 


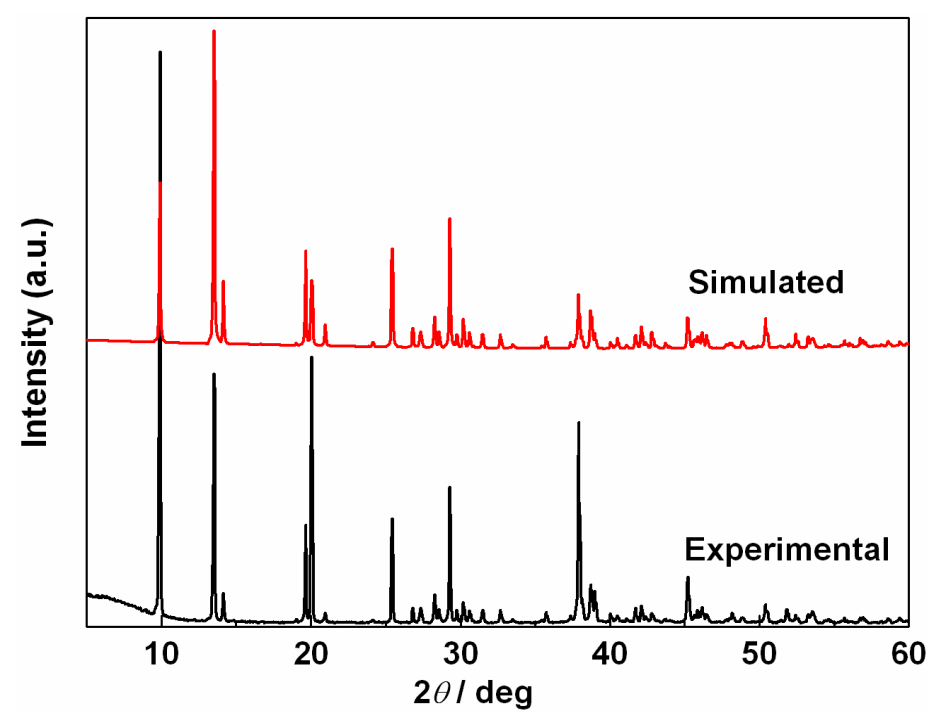

Figure S1. Experimental and simulated PXRD patterns of CFT. The diffraction pattern was recorded on a Bruker D8 Advance diffractometer in Bragg-Brentano geometry using a linear position sensitive detector with $\mathrm{Cu}-K_{\alpha}$ radiation.

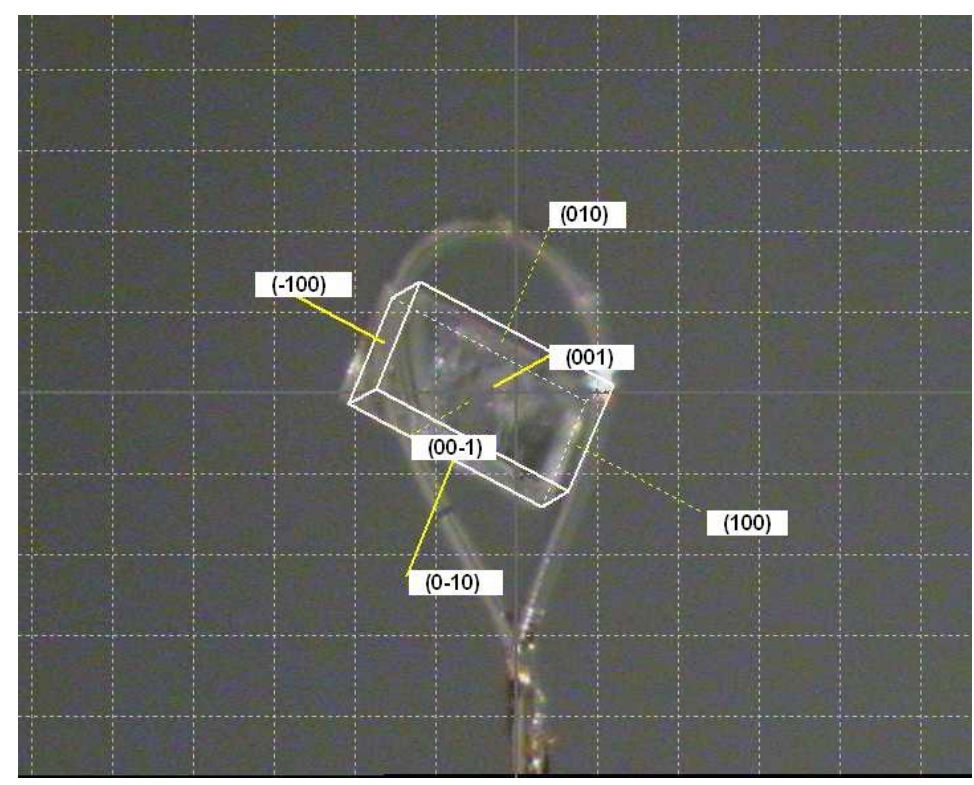

Figure S2. Indexed faces of a typical CFT single crystal. 


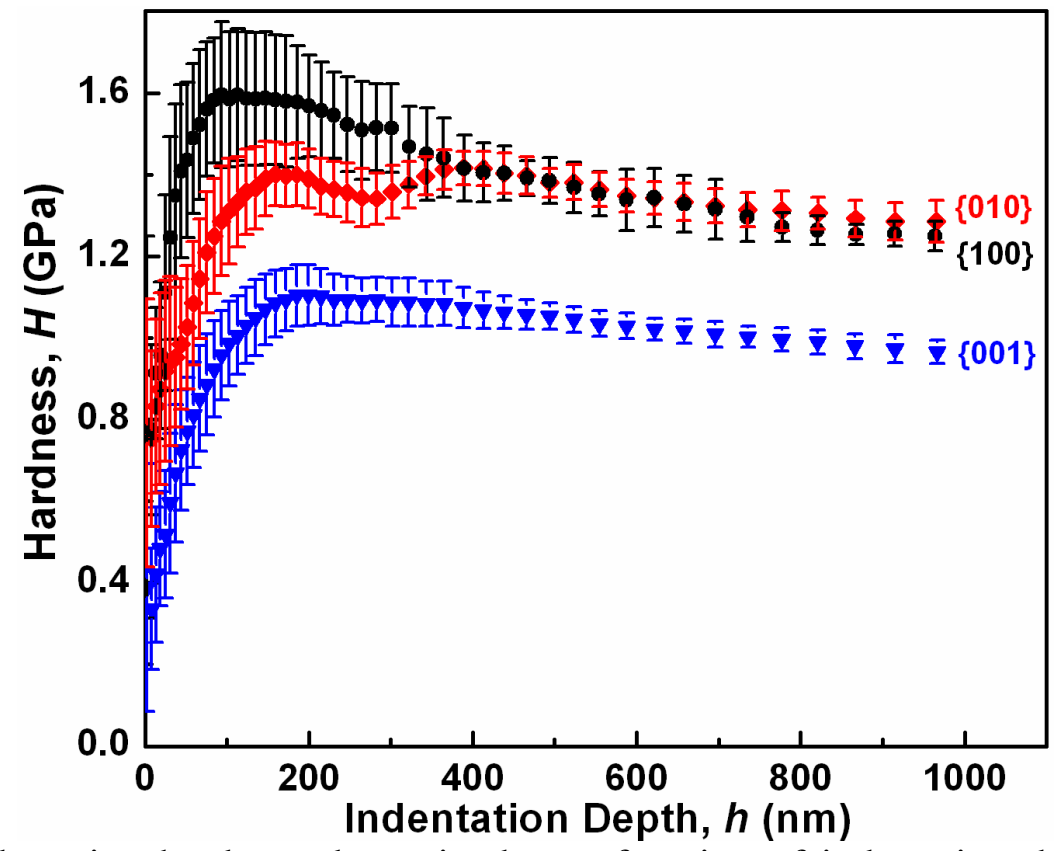

Figure S3. Indentation hardness determined as a function of indentation depth. Error bars represent the standard derivations of 12 measurements for $\{001\}, 13$ measurements for $\{010\}$ and 6 measurements for $\{100\}$.

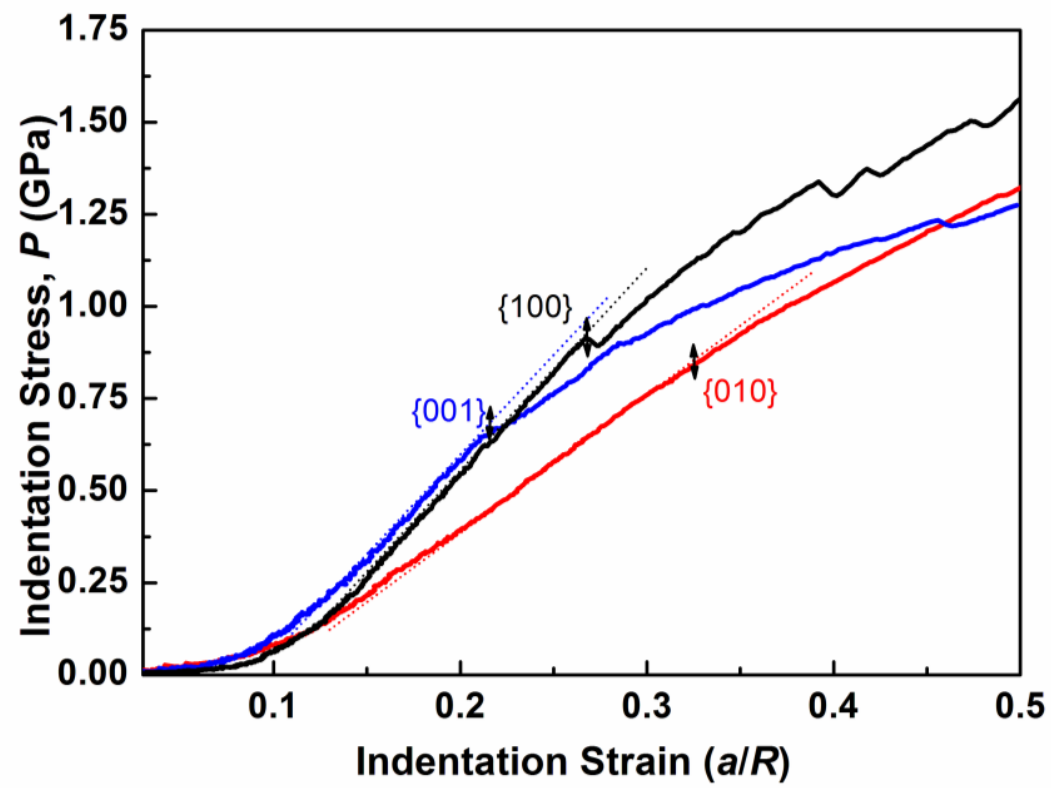

Figure S4. Stress-strain curves of indentation on $\{100\},\{010\}$ and $\{001\}$ respectively. Individual values of the yield stress are obtained from the intersection points of gradient lines in elastic and plastic regions, respectively. 


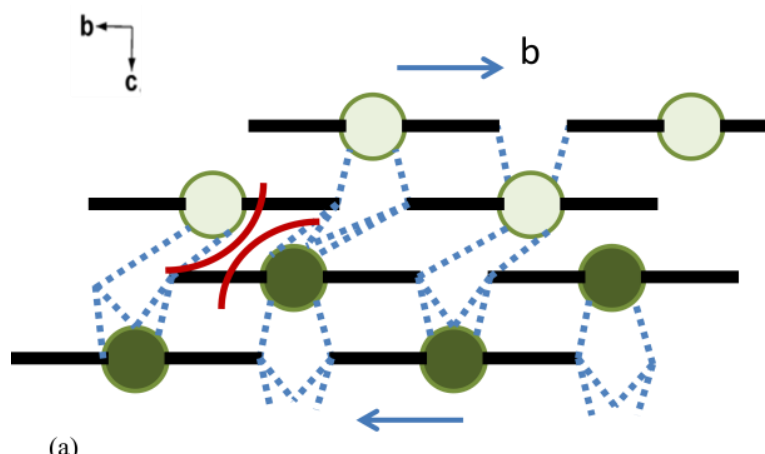

(a)
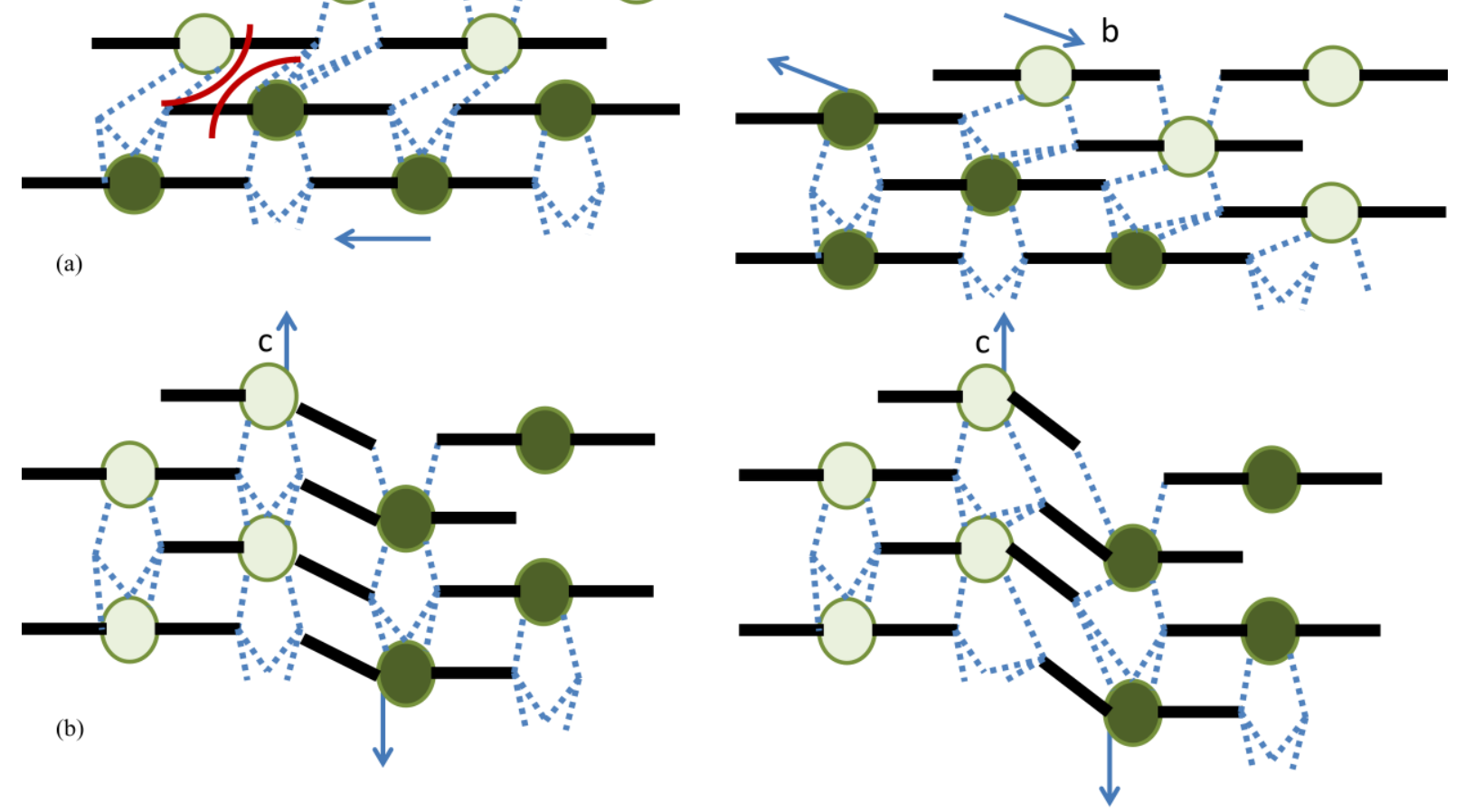

Figure S5. Schematic illustration of plastic deformations via slips of the O-Ca-O inorganic chains (green circles) and the fumarate ligands (black rods) under the indentation tip when indenting along (a) $b$ axis, (b) $c$ axis. Hydrogen bonds are shown as blue dotted lines.

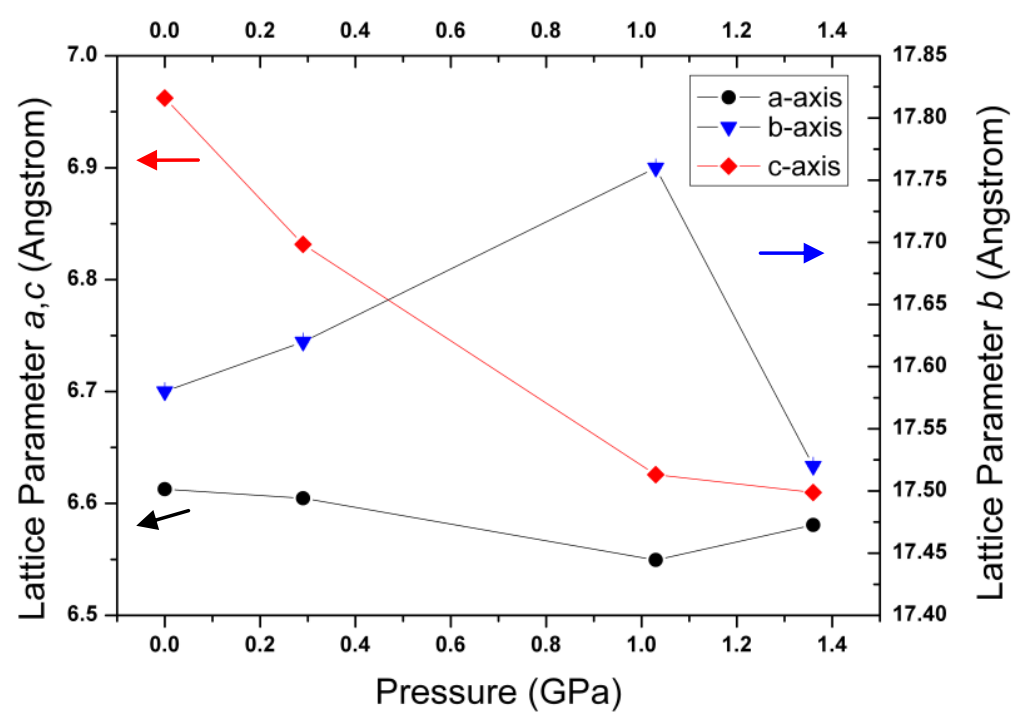

Figure S6. Variations of the lattice parameters as a function of the hydrostatic pressure (negative linear compressibility along $b$ axis seen). 